Journal of Engineering and Applied Sciences 15 (6): 1544-1551, 2020

ISSN: 1816-949X

(c) Medwell Journals, 2020

\title{
Study on Precise Detection Method of Underground Burials using Ground Penetrating Radar
}

\author{
Y. Han \\ Geotechnical Engineering Research Institute, Korean Geotechnical Society, Seoul, South Korea
}

\begin{abstract}
There are many geophysical survey methods using non-destructive rating techniques that can be used to explore underground cavities and ground relaxation zones. However, some geophysical survey methods have factors that affect the characteristics of underground media and the actual soil in the ground contains complex elements that affect geophysical survey. Thus, it is necessary to analyze the effects on geophysical survey methods based on the understanding of soil engineering properties. This study aimed to construct a test bed to identify the accurate location of underground facilities and to conduct an exploration of underground burials using Ground Penetrating Radar (GPR). The study results exhibited that soil stratigraphic classification could be possible and reflected waves were measured at the boundary where the dielectric permittivity was different between an underground burial and the soil. The results of comparison of the actual underground facility location and calculated location from the GPR exploration results showed that similar measurement results could be obtained.
\end{abstract}

Key words: Geophysical survey, GPR (Ground Penetrating Radar) of underground burials, dielectric permittivity, classification, facility

\section{INTRODUCTION}

With the rapid development of urban cities, the development of underground space has increased as well. Due to this development, cavities may be produced by the soil runoff along with groundwater due to the upper soil influx or water leakage around the defects of aging conduits of surrounding underground structures and underground burials by which sudden ground subsidence occurs as a result of the reduction in soil shear strength (Fig. 1).

Studies on underground cavity and burial detection for ground subsidence detection have been actively conducted (Endres et al., 2000; Hagrey and Muller, 2000; Lu and Sato, 2004; Pyke et al., 2008; Kuroda et al., 2009; Kim et al., 2013; Han et al., 2019). In particular, there are GPR exploration, resistivity exploration and surface wave exploration for non-destructive rating techniques that can be used to explore the subsurface. Among them, the GPR exploration technique is an excellent method that is applicable to underground cavity, underground burial and groundwater level observation, bedrock detection, detection of discontinuity such as fragmental zone of fault, etc. and concrete non-destructive rating fields. However, the energy of electromagnetic waves may be weakened by factors (dielectric permittivity, electric conductivity, penetration ratio, etc., depending on underground media) that affect the GPR signals. As described above, geophysical survey methods among non-destructive rating methods are affected by the characteristics of underground media and soils as underground media can be easily separated because soils are discontinued and displacement between particles can easily occur under external force. In addition, the engineering properties of soils are characterized by heterogeneity and aeolotropy which contain various and complex elements depending on the ground depth. Thus, in GPR exploration, penetration of electromagnetic waves
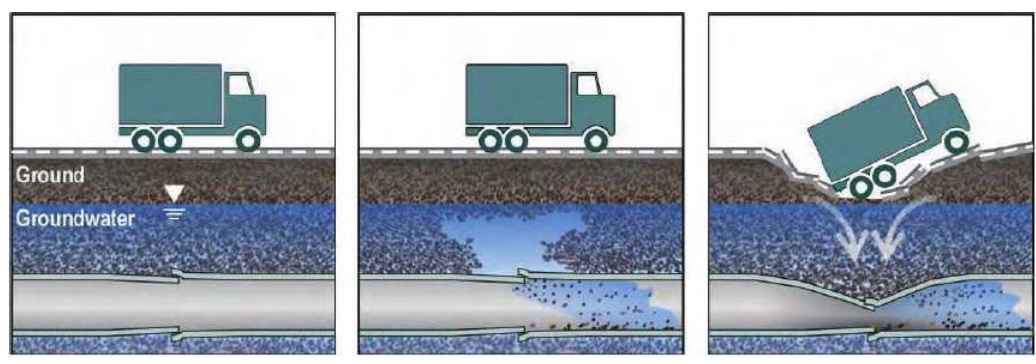

Fig. 1: Schematic diagram of sinkhole occurrence due to aging conduits 
to the deep subsurface is difficult due to severe attenuation of electromagnetic waves if the electric conductivity of the media is high and the penetration depth of the electromagnetic waves may vary according to the frequency as well as being affected complexly by dielectric constant and electric conductivity that the soil has (Cassidy, 2009). This study aims to develop a test bed to consider the various and complex factors in actual grounds, analyze the limitations of the GPR exploration and propose a method of precision detection of underground burials.

\section{MATERIALS AND METHODS}

Theoretical background: GPR surveying is used to examine shallow underground structures using an electromagnetic pulse at frequencies between $10 \mathrm{MHz}-1 \mathrm{GHz}$. This method makes use of electromagnetic waves that are relatively shorter than those used in other survey methods, resulting in a high resolution. In doing so, this method regards measuring and interpreting the reflections and diffractions of the electromagnetic waves according to differences in permittivity of the concerned media to understand geological structures. GRP surveys, in particular have a relatively higher applicability in dry sandstone or conglomerate as such structures allow electromagnetic waves to easily penetrate. On the other hand, this method is subject to large energy losses of the electromagnetic waves when penetrating clay layers due to high conductivity and is not suited to survey such grounds.

The $10 \mathrm{MHz}-1 \mathrm{GHz}$ high-frequency band used in GPR surveys concerns a band in which the displacement current is dominant over the conduction current. In this case, the behavior of the electromagnetic field is governed by the wave equation. The variables of interest with respect to the behavioral properties of the GPR wave concern the rate of attenuation and speed. Assuming a plane electromagnetic wave, the attenuation constant $(\mathrm{a},[\mathrm{dB} / \mathrm{m}])$ and location constant $(\beta,[\mathrm{rad} / \mathrm{m}])$ for the waveband is shown in Eq. 1 and 2:

$$
\begin{aligned}
& a=\frac{\sigma}{2} \frac{\mu}{\varepsilon} \\
& \beta=\omega \mu \varepsilon
\end{aligned}
$$

Where:

$\sigma:$ Conductivity $(\mathrm{S} / \mathrm{m})$

$\varepsilon$ : Permittivity $(\mathrm{F} / \mathrm{m})$

$\mu$ : Permeability $(\mathrm{H} / \mathrm{m})$

$\omega$ : Each frequency

Thus, if the conductivity of the medium is larger or permittivity of the medium becomes smaller, the GPR wave is subject to greater losses irrespective of frequency. However, in the case of high-frequency bands of $100 \mathrm{MHz}$ or higher, due to the relaxation effect, the attenuation constant quickly rises and in turn, rapidly lowers the capable survey depth at which the GPR surveying equipment using frequencies of $100 \mathrm{MHz}$ or higher operate.

Assuming that the permeability of the underground rock is the same as that of a vacuum $\left(\mu=\mu_{0}\right)$, the velocity of the GPR wave (v, [m/nsec]) can be defined as Eq. 3 shown below:

$$
\mathrm{V}=\frac{\mathrm{c}}{\sqrt{\varepsilon \mathrm{r}}}=\frac{0.3}{\sqrt{\varepsilon \mathrm{r}}}
$$

As indicated, the transmission velocity of the GPR wave is unaffected by frequency and depends on permittivity. In this case where $\varepsilon_{\mathrm{r}}=\varepsilon / \varepsilon_{0}$ is relative permittivity, $\mathrm{c}$ is the velocity of the electromagnetic wave, $0.3 \mathrm{~m} / \mathrm{nsec}$ and the wavelength of the GPR wave is shown in Eq. 4:

$$
\lambda=\frac{\mathrm{v}}{\mathrm{f}}=\frac{300}{\varepsilon_{\mathrm{r}} \mathrm{f}}
$$

where, the unit used in is $\mathrm{MHz}$. Due to the relative permittivity of the underground rock being 3 30, the transmission velocity of the GPR wave is $0.01 \sim 0.175 \mathrm{~m} / \mathrm{ns}$. In addition, the relative permittivity of water is 80 which is dramatically larger than that of other substances. Thus, the amount of water content within an underground medium has a dramatic effect on the behavior of radar waves.

There are approximately three factors that drive the attenuation of GPR waves. First, due to the transmission antenna used in GPR surveys being a point source, the waves are transmitted at a $90^{\circ}$ angle in the form of a cone from its transmission source. Therefore, as the distance from the transmission antenna increases, the size of the signal attenuates at a rate of $1 / \mathrm{r}$. Second, as some energy converts to heat according to the attenuation constant, the signal attenuates. This is called absorption. Third, energy loss occurs at boundaries as the GPR waves reflect and penetrate. Assuming vertical propagation, the reflection coefficient is shown in Eq. 5:

$$
\mathrm{k}=\varepsilon_{1}-\frac{\varepsilon_{2}}{\varepsilon_{1}}+\varepsilon_{2}
$$

where, $\varepsilon_{1}, \varepsilon_{2}$ is the relative permittivity of both sides of the boundaries. Thus, in locations where a large amount of various geological noises is present (including micro-inhomogeneities), the applicable depths of GPR surveying become reduced. 
J. Eng. Applied Sci., 15 (6): 1544-1551, 2020

Table 1: Relationship with variables of underground electrical characteristics, GPR exploration and frequency

\begin{tabular}{|c|c|c|c|c|c|c|c|c|c|}
\hline \multirow[b]{2}{*}{ Measurement variable } & \multicolumn{3}{|c|}{ Permittivity } & \multicolumn{3}{|c|}{ Conductivity } & \multicolumn{3}{|c|}{ Frequency } \\
\hline & Low & $\rightarrow$ & High & Low & $\rightarrow$ & High & Low & $\rightarrow$ & High \\
\hline Velocity of electromagnetic wave & Fast & $\rightarrow$ & Slow & & & & & & \\
\hline Attenuation & High & $\rightarrow$ & Low & Low & $\rightarrow$ & High & Low & $\rightarrow$ & High \\
\hline Depth of investigation & Shallow & $\rightarrow$ & Deep & Deep & $\rightarrow$ & Shallow & Deep & $\rightarrow$ & Shallow \\
\hline Wavelength & Long & $\rightarrow$ & Short & & & & Long & $\rightarrow$ & Short \\
\hline Resolution & Low & $\rightarrow$ & High & & & & Low & $\rightarrow$ & High \\
\hline
\end{tabular}
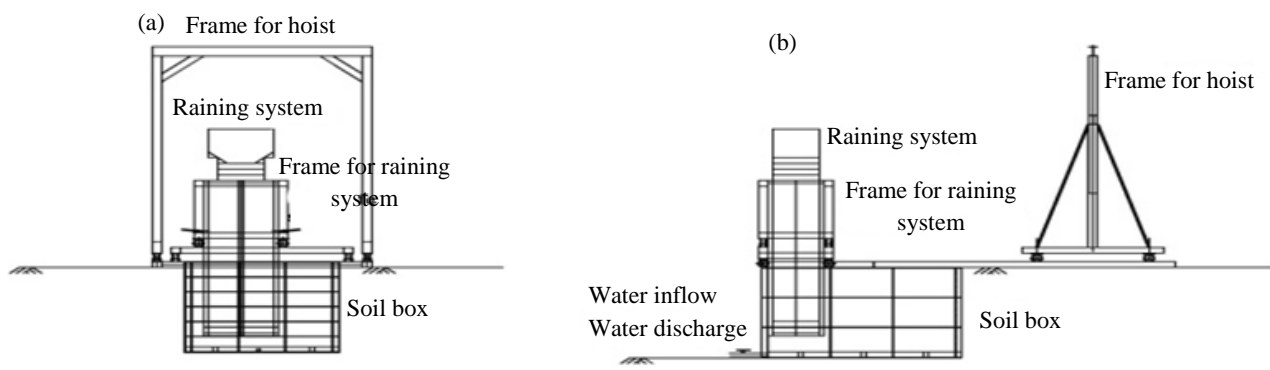

Fig. 2(a, b): Conceptual diagram of scale model test (Han, 2020), (a) Front view and (b) Side view

GPR resolution regards the capacity to distinguish between two reflected signals that are temporally adjacent. Therefore, resolution is a function of frequency. The transmitting and receiving antenna used in GPR surveys are created to emit signals within a certain frequency band and this frequency band is known as the bandwidth of the antenna. In addition, the frequency that presents the greatest reaction is known as the center frequency. In light of this, it is thus the case that antennas have a unique center frequency and that most GPR surveying equipment are designed to have the same bandwidth as the center frequency.

The minimal detectable object size is referred to as the 'resolution and differs according to the Earth. Resolution also refers to half of the length of one wave $\left(\lambda_{1}\right)$. The center frequency must become larger to enhance resolution. Despite resolution enhancement as a result of increasing center frequencies, this results in greater attenuation in GPR surveys and lowered applicable survey depths. Therefore, it is advantageous to prioritize applicable survey depths when selecting antennas rather than focusing on resolution. This is recommended if there is no information regarding the approximate depth or permittivity of the object to be surveyed. Due to the velocity of the GPR wave being a function of permittivity, resolution changes according to media must be considered. The lower limit of the frequency is determined by resolution and the upper limit is determined according to the applicable survey depth and geological noise (Table 1).

\section{Response analysis of GPR exploration through model experiments}

Construction of scale model: A scale model was created as shown in Fig. 2 to examine the various and complex factors of the Earth and to measure the electrical properties of the Earth. The scale model was created using acrylic boards to form a chamber measuring a length of $2.1 \mathrm{~m}$, a width of $1.5 \mathrm{~m}$ and a height of $1.5 \mathrm{~m}$. To adjust the looseness or denseness of the sand, a mobile frame installed with a sand showering device capable of forward, backward and side-to-side movement was installed on top of the scale model. The height at which sand fell from this contraption was made to be adjustable. In addition, a water inflow and discharge mechanism was installed on the bottom of the scale model to allow groundwater levels of the formed sand grounds to be adjustable (Fig. 2).

To analyze the response of the GPR in the sand ground assembled using the scale model, Styrofoam $(0.3 \times 0.15 \mathrm{~m})$ whose dielectric constant was the same with that of air was buried ata height of $0.9 \mathrm{~m}$ from the bottom as shown in Fig. 3 and the GPR exploration with $800 \mathrm{MHz}$ frequency was conducted at each of line-1-9 with $0.15 \mathrm{~m}$ gaps as shown in Fig. 3b. The specimens used in the experiment were a mixed soil of $96 \%$ sand and $4 \%$ silt and its coefficient of uniformity and coefficient of curvature were 4.38 and 1.63, respectively. The specimens used in the experiment were classified using the Unified Soil Classification System (USCS) and the specimens were classified down to poorly graded sand (SP) whose grain size distribution was poor.

GPR exploration and analysis of the results: GPR exploration was conducted at a total of nine sides from line-1-9 with gaps of $0.15 \mathrm{~m}$ using an $800 \mathrm{MHz}$ antenna as shown in Fig. $3 \mathrm{~b}$ to analyze the response of the GPR. The results of GPR exploration exhibited a clear reflection pattern showing a convex hyperbolic shape above the buried cavity in the line-3-7 sections as shown in Fig. 4 
(a)

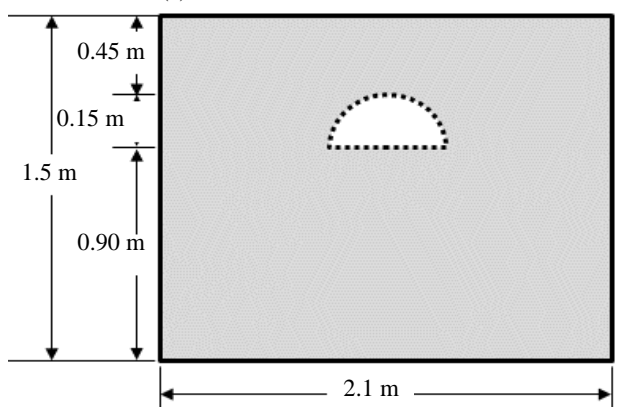

(b)

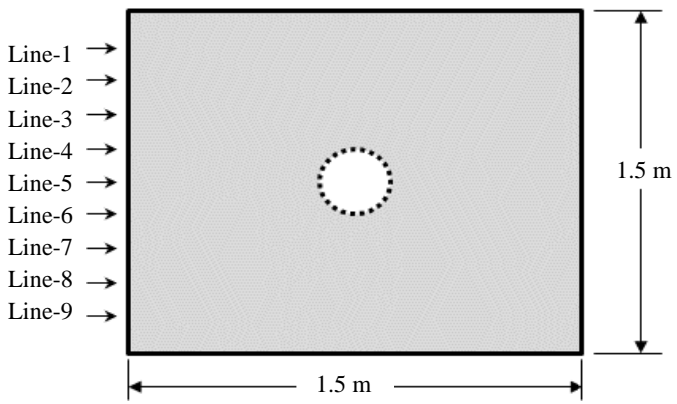

Fig. 3(a, b): Layout of Styrofoam for GPR exploration, (a) Front view and (b) Side view

(a)

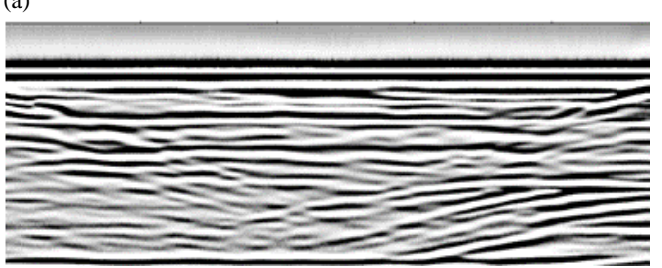

(c)

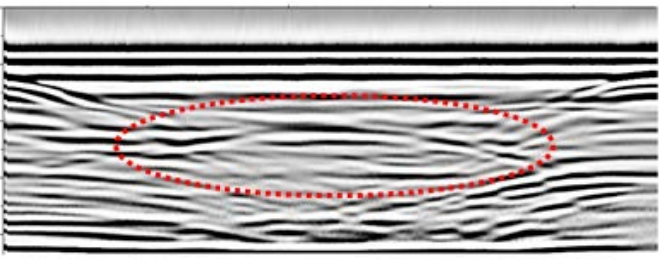

(e)

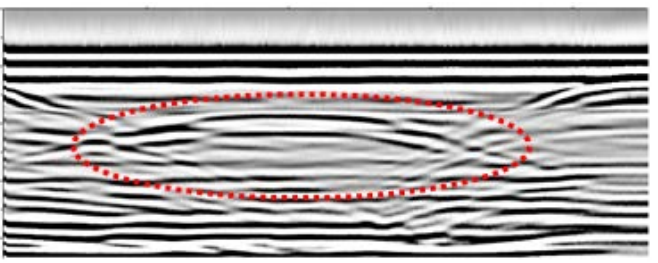

(g)

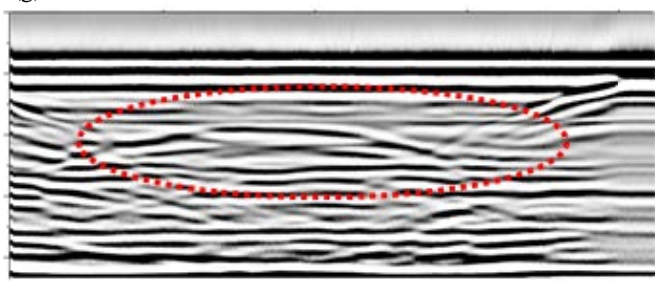

(b)

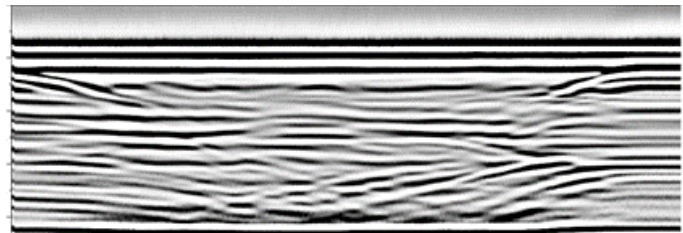

(d)

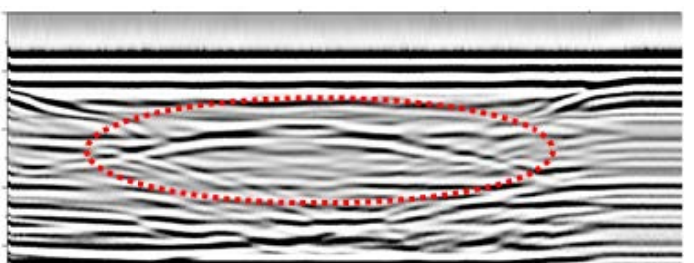

(f)

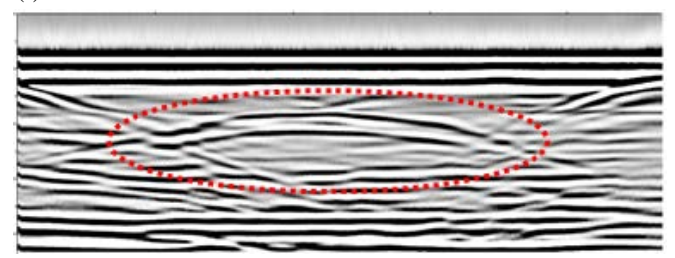

(h)

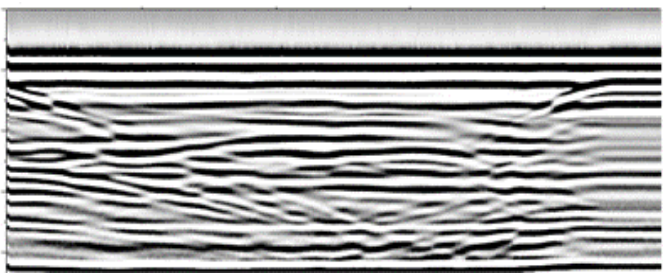

(i)

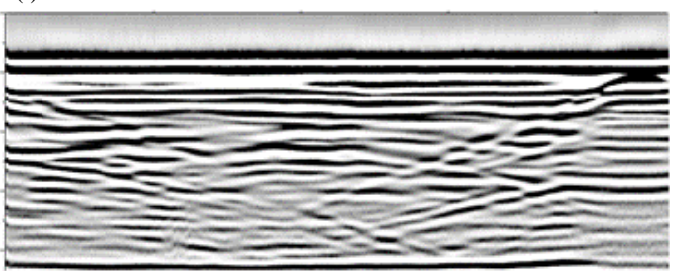

Fig. 4(a-i): Exploration results of GPR (exploration section: Line-1 to Line-9) 
J. Eng. Applied Sci., 15 (6): 1544-1551, 2020

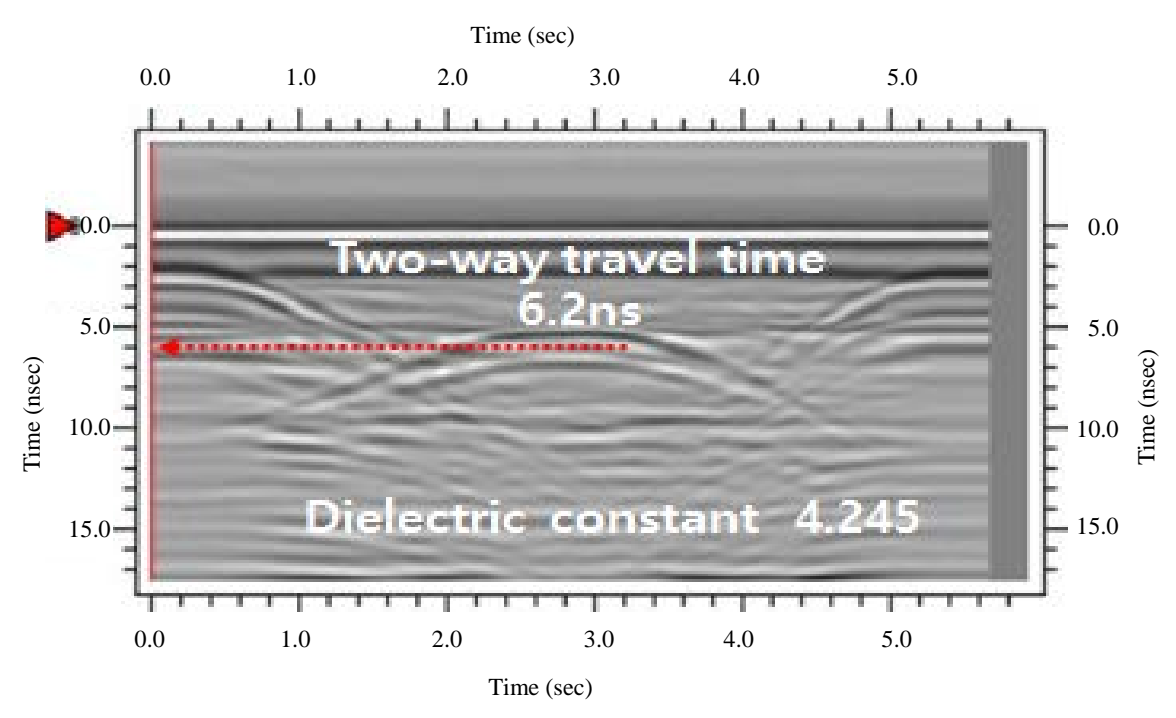

Fig. 5: GPR exploration result of line-5

and a $0.3 \mathrm{~m}$ cavity size was identifiable at a depth of $0.45 \mathrm{~m}$. However, no response to the buried cavity was revealed at the line- 1 and 2 or line- 8 and 9 sections.

To accurately identify the location information of the burial from the GPR exploration results, the time section should be converted to the depth section in the GPR exploration data. Since, GPR records the penetrated and reflected waves from the boundary surface where the physical properties of the medium change after the transmission source of electromagnetic waves is penetrated, it is critical to have information about the propagation velocity distribution of the electromagnetic waves. Equation 6 can be used to convert a two-way travel time of the electromagnetic wave into a depth:

$$
d=\sqrt{\left(\frac{v \times t}{2}\right)^{2}-\left(\frac{x}{2}\right)^{2}}
$$

Where:

d : Depth (m)

$\mathrm{v}$ : Velocity of electromagnetic wave $(\mathrm{m} / \mathrm{sec})$

$\mathrm{t}$ : Two-way travel time (sec)

$\mathrm{x}$ : The distance between antennas $(\mathrm{m})$

Although, velocity estimated based on the ground conditions, it can be used without any significant problem and it is preferable to measure propagation velocity at the site through common transmission points or various channel explorations, etc. If it is impossible to measure propagation velocity of electromagnetic wave at the site during exploration or if estimating propagation velocity is difficult from the data obtained through exploration, the propagation velocity of electromagnetic wave can be calculated by measuring and using the dielectric permittivity of the ground:
Table 2: Dielectric constant and conductivity of sand (Han, 2020)

\begin{tabular}{lcc}
\hline Conditions of sand & Dielectric constant & Conductivity $(\mathrm{mS} / \mathrm{m})$ \\
\hline Loose sand $(\mathrm{Dr}=40 \%)$ & 3.451 & 0.1 \\
Dense sand $(\mathrm{Dr}=60 \%)$ & 4.245 & 0.1 \\
Saturated sand & 18.690 & 9.6 \\
Unsaturated sand & 7.813 & 4.5 \\
\hline & \\
$\qquad$ \\
\\
$\mathrm{V}=\frac{\mathrm{C}}{\sqrt{\varepsilon_{\mathrm{r}}}}$
\end{tabular}

For the sands used in the scale model in this study, Han (2020) defined the correlation between dielectric constant and electric conductivity according to the characteristics of the sands as presented in Table 2.

The dielectric constant $\left(\varepsilon_{\mathrm{r}}=4.245\right)$ measured in the experiment was substituted to Eq. 7 to calculate the electromagnetic velocity which was then substituted to Eq. 6 to calculate the two-way travel time of the reflected wave from the boundary where the media are different at the $0.45 \mathrm{~m}$ location which was $6.2 \mathrm{nsec}$. The results of analysis of the line-5 GPR exploration section in Fig. 5 showed that the GPR exploration waveform due to the burial cavity was an upper convex hyperbolic shape. The two-way travel time of the hyperbolic center was measured and the result was 6.25 nsec which was the same value as the calculation time.

\section{RESULTS AND DISCUSSION}

Analysis on the GPR exploration response for underground burial exploration below the asphalt pavement 

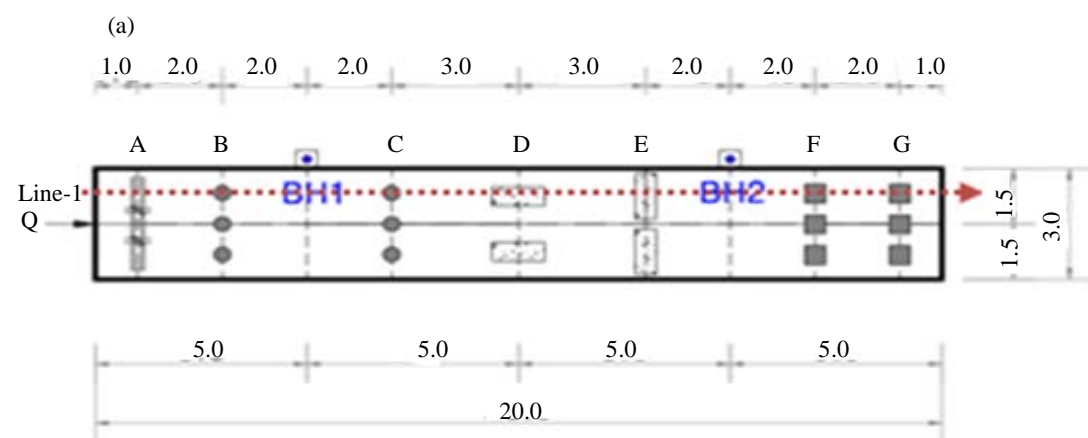

No. $1+5$

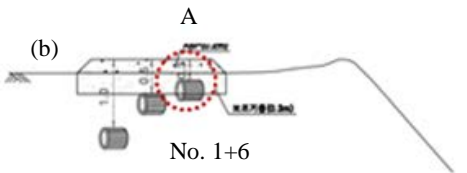

B
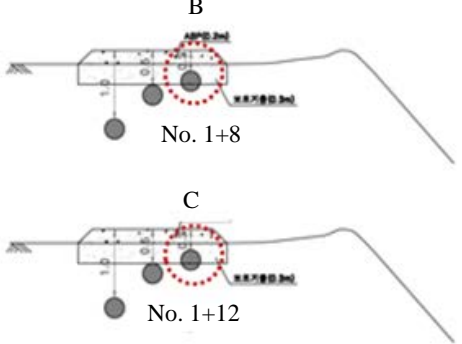

$\mathrm{D}$

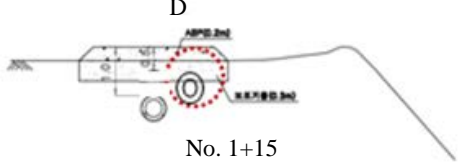

No. $2+5$

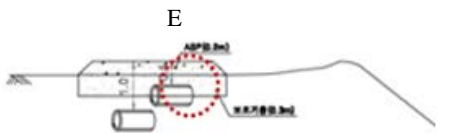

No. $1+18$
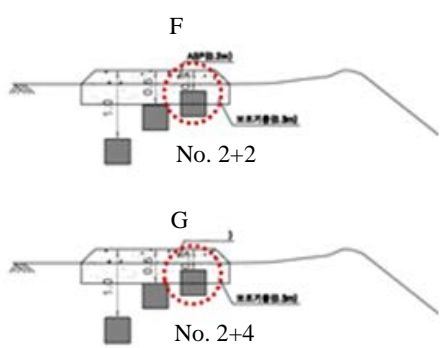

Fig. 6(a, b): Test bed for exploration, (a) Plan view and (b) Cross-sectional view

Development of the test bed: The test bed used in this study is shown in Fig. 6 as follows: Polyvinyl Chloride (PVC) corrugated tube $(\varphi=0.3 \mathrm{~m}, \mathrm{~L}=2.5 \mathrm{~m})$ and a model $(\mathrm{D}=0.4,0.5 \times 0.5 \mathrm{~m})$ made of Styrofoam $(1.03$ of dielectric constant) whose dielectric constant was relatively similar to that of air (1.0005) were buried at the depths of $0.3,0.5$ and $1.0 \mathrm{~m}$, respectively, below the ground and concrete hume pipes $(\varphi=0.5 \mathrm{~m}, \mathrm{~L}=2.5 \mathrm{~m})$ were buried at the depths of $0.5 \mathrm{~m}$ and $1.0 \mathrm{~m}$ below the ground. The thickness of the asphalt pavement was $0.2 \mathrm{~m}$ and the subbase thickness was $0.3 \mathrm{~m}$. The measured GPR exploration section for underground burial exploration was line-1 in Fig. 6a. In the line-1 A section, a PVC corrugated tube was buried at a depth of $0.3 \mathrm{~m}$ and in the $\mathrm{B}$ and $\mathrm{C}$ sections, a circular shape made of Styrofoam was buried at a depth of $0.3 \mathrm{~m}$. In the $\mathrm{D}$ section, a concrete hume pipe was buried at a depth of $0.5 \mathrm{~m}$ in the longitudinal direction and in the $\mathrm{E}$ section, a concrete hume pipe was buried at a depth of $0.5 \mathrm{~m}$ in the transverse direction. In the $\mathrm{F}$ and $\mathrm{G}$ sections, a rectangular shape made of Styrofoam was buried at a depth of $0.3 \mathrm{~m}$.
GPR exploration and analysis of the results: To analyze the response of the GPR, GPR exploration was conducted at the line-1 exploration section in Fig. 6a using a $400 \mathrm{MHz}$ antenna. As a result, a waveform curve size differed according to the media whose dielectric permittivity was different as shown in Fig. 7 and the diffracted wave (hyperbola) was clearly seen. To calculate the accurate location of the underground burial according to a medium, the electromagnetic velocity that was calculated by substituting the dielectric constant $\left(\varepsilon_{\mathrm{r}}=4.245\right)$ of the assembled sand in Table 2 to Eq. 7 was substituted to Eq. 6. Accordingly, the calculated result showed that when the two-way travel time of the reflected wave to the receiver was 4.2 nsec, the wave was reflected at the boundary where the media were different at a depth of $0.3 \mathrm{~m}$. In addition, when the two-way travel time was $7.0 \mathrm{nsec}$, the calculated result showed that the wave was reflected at the boundary where the media were different at a depth of $0.5 \mathrm{~m}$. The line- 1 exploration section in Fig. 6a revealed that the underground burial depth and the GPR exploration results were at the same location. 


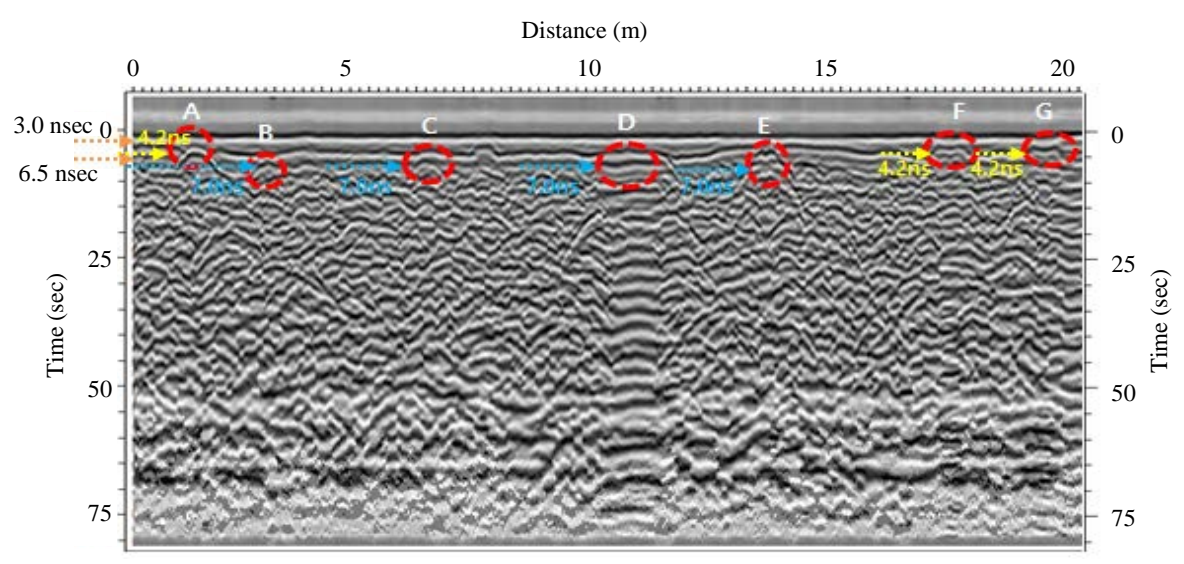

Fig. 7: GPR exploration results

However, the GPR exploration results in the B and C sections showed a response of cavity at a depth of $0.5 \mathrm{~m}$. This indicated that multi-reflection signals below the reflection surface of the cavity and asphalt whose media were different were measured at a similar location.

The stratigraphic structure whose media were different could also be identified based on the GPR exploration results. The stratigraphic distinction between the lower asphalt and upper subbase was clearly seen at the place whose two-way travel time was 3.0 nsec. Normally, the dielectric constant of asphalts was ranged from 3-5 (Kim et al., 2013). When a two-way travel time of reflected wave to the receiver was $3.0 \mathrm{nsec}$, the wave was reflected at the boundary whose media were different at an approximate depth of $0.2 \mathrm{~m}$. In addition, stratigraphic distinction at the lower subbase was also clearly seen. However, the thickness of the subbase was found to be inconsistent which was due to the effect of lift thickness and compaction during construction.

\section{CONCLUSION}

The concrete underground burials and underground cavity were explored using GPR exploration in this study and the following conclusions were made: waveform size and shape differed according to the media whose dielectric permittivity was different and the diffracted wave was clearly seen at the boundary whose dielectric permittivity was different from that of soil. The GPR exploration waveform due to the burial cavity showed a reflection pattern of an upper convex hyperbolic shape and a two-way travel time that corresponded to the center of the hyperbola should be measured at the reflected boundary.

The exploration results using the center frequency of $800 \mathrm{MHz}$ showed that a $0.3 \mathrm{~m}$ hemispherically shaped cavity could be identified around $0.5 \mathrm{~m}$ below the ground from the sandy ground without underground water and it could be detected up to the $0.3 \mathrm{~m}$ range from the cavity center. The exploration results using the center frequency of $400 \mathrm{MHz}$ showed that underground burials and cavities up to $0.5 \mathrm{~m}$ in size located at an approximate depth of $0.5 \mathrm{~m}$ below the concrete paved road could be clearly identified.

\section{ACKNOWLEDGEMENTS}

This research was supported by the Korea Agency for Infrastructure Technology Advancement under the Ministry of Land, Infrastructure and Transport of the Korean Government. (Project Number: 19SCIP-B10815305).

\section{REFERENCES}

Cassidy, N.J., 2009. Electrical and Magnetic Properties of Rocks, Soils and Fluids. In: Ground Penetrating Radar: Theory and Applications, Jol, H.M. (Ed.). Elsevier, Amsterdam, Netherlands, ISBN: 978-0-444-53348-7, pp: 41-67.

Endres, A.L., W.P. Clement and D.L. Rudolph, 2000. Ground penetrating radar imaging of an aquifer during a pumping test. Groundwater, 38: 566-576.

Hagrey, A. and C. Muller, 2001. GPR study of pore water content and salinity in sand. Geophys. Prospect., 48: 63-85.

Han, Y., 2020. A study on the limitations of GPR exploration in sand layer through analysis of the ground subsidence mechanism. J. Eng. Applied Sci., 15: 319-326.

Han, Y., J. Kim, H. Jang, H. Sohn and K. Yoo, 2019. A case study of the ground collapse mechanism due to excavation-induced groundwater level disturbance. J. Eng. Applied Sci., 14: 8676-8685. 
Kim, B.W., H.S. Kim, D.H. Choi and Y.K. Koh, 2013. Estimation of groundwater table using Ground Penetration Radar (GPR) in a sand tank model and at an Alluvial field site. J. Eng. Geol., 23: 201-216.

Kuroda, S., H. Jang and H.J. Kim, 2009. Time-lapse borehole radar monitoring of an infiltration experiment in the vadose zone. J. Applied Geophys., 67: 361-366.
Lu, Q. and M. Sato, 2004. Estimation of hydraulic property of an unconfined aquifer by GPR. Proceedings of the 10th International Conference on Grounds Penetrating Radar (GPR'04), June 21-24, 2004, IEEE, Delft, The Netherlands, pp: 715-718.

Pyke, K., S. Eyuboglu, J.J. Daniels and M. Vendl, 2008. A controlled experiment to determine the water table response using ground penetrating radar. J. Environ. Eng. Geophys., 13: 335-342. 